\title{
Ökodumping : Mythos oder Realität
}

Das Beispiel der schweizerischen Direktinvestitionen

Thomas Straubhaar et Markus Wyss

\section{(2) OpenEdition}

Journals

Édition électronique

URL : http://journals.openedition.org/sjep/1426

DOI : $10.4000 /$ sjep.1426

ISSN : 1663-9677

Éditeur

Institut de hautes études internationales et du développement

Édition imprimée

Date de publication : 1 janvier 1994

Pagination : 251-267

ISSN : 1660-5926

Référence électronique

Thomas Straubhaar et Markus Wyss, « Ökodumping : Mythos oder Realität », Schweizerisches Jahrbuch für Entwicklungspolitik [En ligne], 13 | 1994, mis en ligne le 10 juin 2013, consulté le 08 septembre 2020. URL : http://journals.openedition.org/sjep/1426 ; DOI : https://doi.org/10.4000/sjep. 1426 


\title{
Ökodumping: Mythos oder Realität
Das Beispiel der schweizerischen Direktinvestitionen
}

\author{
Thomas Straubhaar, Markus Wyss*
}

\section{Einleitung}

\subsection{Motivation}

Im Zuge der Globalisierung der Weltwirtschaft einerseits und den weltweit wachsenden Umweltproblemen andererseits stellt sich die Frage nach der Rolle der multinationalen Konzerne im Prozess einer "nachhaltigen Entwicklung". Unternehmen reagieren auf die Globalisierung der Märkte mit einer zunehmenden Internationalisierung ihrer Informations-, Finanz-, Investitions-, Produktions- und Absatzaktivitäten. Mit dieser Internationalisierungsstrategie streben die Unternehmungen eine effiziente Kombination von firmen-, transaktions- und länderspezifischen Faktoren an (1).

Die Standortwahl eines Industriebetriebes wird von einer Vielzahl physischer, ökonomischer, sozialer, politischer und kultureller Bedingungen beeinflusst. Verlagern multinationale Unternehmungen im Zuge ihrer Faktorallokation jene Produktionsschritte, welche die Umwelt besonders belasten an Produktionsstandorte mit geringeren umweltgesetzlichen Regulierungsvorschriften - was wir in der Folge "Standortarbitrage" nennen wollen - oder produzieren sie im Sinne einer "One Standard Strategie" weltweit mit den gleichen Umweltstandards, womit nicht

* Wir danken Rainer Durth, Reinhard Felke und Petra Becker vom Europa-Kolleg in Hamburg für die wertvollen Hinweise. 
zuletzt der Transfer von Umweltschutztechnologien von Nord nach Süd aktiv gefördert würde? (2)

In den achtziger Jahren führte die Analyse dieser Fragestellung zu kontroversen Resultaten. Während einige Studien von einer empirischen Evidenz einer Standortarbitrage von amerikanischen und japanischen Firmen sprechen (3), verneinen andere theoretische und empirische Studien die Existenz einer ökologisch motivierten Produktionsverlagerung (4). Letztere argumentieren unter anderem mit den hohen Transaktionskosten (Transport, Handelshemmnisse etc.), die durch eine Produktionsverlagerung entstehen und damit die eingesparten Umweltschutzkosten kompensieren. Teile der Transaktionskosten könnten sich im Zuge der weltweiten Handelsliberalisierungen im Zusammenhang mit der UruguayRunde des GATT verringern. Unter diesen Vorzeichen sind wohl auch einige der jüngsten Studien, die sich mit der Frage der Standortarbitrage befassen, entstanden (5).

\subsection{Zielsetzung}

In unserer empirischen Untersuchung gehen wir auf die umweltpolitisch bedingte Faktorallokation wichtiger multinationaler Unternehmungen mit Hauptsitz in der Schweiz ein. Hierbei legen wir das Schwergewicht auf die empirische Untersuchung mikroökonomischer Reaktionsweisen. Ökonomisches Prozessverständnis und ökologische Wirkungsanalyse wurden interdisziplinär aggregiert. Insbesondere stehen folgende Fragen im Zentrum unserer Analyse (6).

1. Wieweit beeinflussen Unterschiede in der Umweltgesetzgebung zwischen den weniger entwickelten Staaten und der Schweiz die Standortwahl eines Unternehmens? Kommt es zu einer Standortarbitrage aufgrund unterschiedlicher gesetzlicher Umweltvorschriften in verschiedenen Ländern? Nutzen schweizerische Firmen die Standorte mit geringer staatlicher Aufsicht zu umweltbelastender Produktion?

2. Verfolgen schweizerische Firmen bezüglich des Umweltschutzes eine 'One Standard Strategie'? Produzieren Firmen in weniger entwickelten Ländern nach den gleichen ökologischen Grundsätzen wie in der Schweiz?

3. Welche Mehrkosten entstehen, wenn eine Produktionsstätte in einem weniger entwickelten Land nicht mit den lokal üblichen Umweltstandards gebaut und betrieben wird, sondern mit den Umweltnormen, die in der Schweiz gelten?

Normative Fragen wurden bewusst ausgeklammert wie etwa die Frage, ob eine Produktionsverlagerung aus Gründen unterschiedlicher Umweltstandards unternehmensethisch vertretbar sei oder ob Produktionsverlagerungen im Sinne eines globalen Immissionsausgleichs gar gefördert werden müssten.

\subsection{Abgrenzung, theoretische Grundlagen}

Um unerwünschte Ineffizienz zu verhindern oder Innovationen zu fördern kann der Staat durch administrative Regulierungen ausgewählte Produktionsfaktoren 
künstlich beschränken. Solche Massnahmen sind aus der Sicht der Staaten dann besonders sinnvoll, wenn sie nach dem Prinzip der "ausgewählten Benachteiligung" gewisser Produktionsfaktoren (selective factor disadvantage) eine Entwicklung vorwegnehmen, die ohnehin früher oder später erwartet werden muss; Dann nämlich erweisen sich kurzfristige statische Nachteile längerfristig als dynamische komparative Vorteile (7). So kann zum Beispiel durch eine umweltpolitische $\mathbb{U}^{*}$ Massnahme Wasser derart verteuert werden, dass ein Produktionsstandort bei 9 einer gegebenen wasserintensiven Produktionsmethode im Verhältnis zu anderen de leaw Standorten benachteiligt wird. Ein Betrieb kann auf zwei Arten reagieren: Er kann den Standort wechseln oder aber neue, weniger wasserintensive Produktionsmethoden entwickeln, die es erlauben, die Faktoreffizienz zu erhöhen und damit Kosten zu senken (8).

Für unsere Untersuchung ist die Frage nach dem Einbezug der Umweltgesetzgebung in die Standortwahl eines Unternehmens wichtig. Strenge umweltpolitische Vorschriften oder ein grosses Umweltbewusstsein der Bevölkerung haben für eine industrielle Unternehmung hauptsächlich in drei Bereichen Folgen:

1. Steigerung der Investitions- und Betriebskosten,

2. Verzögerung und Erschwerung von Bewilligungen für neue Produktionsstandorte,

3. Verzicht auf gewisse Rohstoffe, Produktionsmethoden oder Produkte.

Soweit institutionelle Voraussetzungen national unterschiedlich ausfallen, kann potentiell ein Anreiz entstehen, Standorte mit "günstigen" solchen mit "weniger günstigen" Bedingungen vorzuziehen. Eine besondere Form dieser Standortwahl ist die ökologisch bedingte "Standortarbitrage" (9). Der Produktionsfaktor Umwelt als Lieferant von Rohstoffen und als Empfänger von Abfällen wird dort genutzt, wo er am billigsten ist. Am billigsten ist er meist in jenen Ländern, die keine Umweltschutzgesetzgebung kennen oder in denen der Vollzug von Umweltschutzvorschriften ungenügend ist. In einigen empirischen Untersuchungen wird gezeigt, dass - vor allem japanische und amerikanische - Unternehmen aufgrund der immer strenger werdenden Umweltgesetze in den industrialisierten Ländern ihre besonders umweltbelastenden Produktionsbereiche in Entwicklungsländer verlagern (10).

In der Industrieökonomie hat die Umwelt als Produktionsfaktor folgende hauptsächliche Funktionen (11):

1. Sie stellt dem Produktionsprozess erneuerbare und nichterneuerbare Ressourcen zur Verfügung (Inputfunktion).

2. Sie nimmt aus demselben Produktionsprozess die Abfälle und Emissionen auf (Outputfunktion) und bereitet diese zum Teil wieder auf (Regenerationsfunktion).

Aus historischen Gründen sind die mikroökonomischen Kosten zur Nutzung von Boden, Wasser und Luft niedriger als die gesamtwirtschaftlichen Kosten, die 
durch die Nutzung dieser Faktoren entstehen (12). Üblicherweise wird versucht, durch umweltpolitische Massnahmen die externen Kosten der Umweltnutzung zu internalisieren (13). Interessant ist nun die aussenwirtschaftliche Relevanz solcher Massnahmen. Werden umweltpolitische Instrumente multilateral ausgehandelt und in allen Staaten eingeführt, dann sind sie aussenwirtschaftlich neutral (14). Werden aber Umweltregulierungen (explizit oder implizit) von einem Staat isoliert so festlegt, dass die Grenzkosten der Umweltbelastung - im Gegensatz zu anderen Staaten - nicht voll internalisiert werden müssen, wird von Öko-Dumping gesprochen (15).

Im Zusammenhang mit der nationalen schweizerischen Umweltpolitik stellt sich folgende Frage: Führt die selektive Begrenzung von Umweltfaktoren durch die schweizerische Umweltpolitik auch bei schweizerischen Firmen zu Standortverlagerungen von Produktionskapazitäten mit hoher Umweltintensität, oder werden nicht vielmehr diese Firmen zu Innovationen im Bereich der Umwelttechnologie und damit zu einer effizienteren Nutzung der knappen Umweltgüter angespornt? Die vorliegende Studie beschränkt sich auf Industrieprojekte im Ausland, deren Erfolg von schweizerischem Kapital oder von schweizerischem ManagementKnow-how in entscheidendem Masse mitbestimmt wird (16). Im weiteren konzentrieren wir uns explizit auf multinationale Unternehmungen. Innerhalb des Wirtschaftskreislaufes liegt das Hauptgewicht unserer Studie bei der Produktion von Gütern. Ausgeklammert wird hingegen die Umweltrelevanz des Konsums und der Entsorgung dieser Produkte (17).

\section{Untersuchungsmethode}

Unsere Untersuchung basiert auf einer Analyse von Fallstudien. Im Rahmen des nationalen Forschungsprogramms $\mathrm{Nr}$. 28 des Schweizerischen Nationalfonds zur Förderung wissenschaftlicher Forschung wurden in den Jahren 1990 und 1991 Daten erarbeitet einerseits mittels einer Dokumentenanalyse und andererseits mit standardisierten Interviews während zweier Feldaufenthalte. Durch Geschäftsberichte und offizielle Publikationen der Konzerne zum Umweltschutz entstand in einer ersten Phase ein Bild der Rolle, welche die Konzernleitungen und die ausländischen Töchter dem Umweltschutz auf der strategischen Ebene zuerkennen. Dieses Bild wurde vervollständigt durch Gespräche mit Mitgliedern der Konzernleitungen und deren Umweltbeauftragten am Hauptsitz der Firmen. In zwei Feldaufenthalten (im Winter 1990 in Indien und im Sommer 1991 in Brasilien) wurden mit der Methode von kurzen Umweltaudits die Umweltschutzmassnahmen von Produktionsbetrieben besichtigt und standardisierte Interviews durchgeführt. Interviewpartner waren die Werksleiter, deren Umweltbeauftragte und oft zusätzlich Mitglieder der Unternehmensleitung. Zur Überprüfung der Resultate fanden in beiden Ländern Gespräche mit Mitgliedern der Umweltschutzbehörden auf der nationalen, der provinzialen und der kommunalen Ebene sowie mit Vertretern von Umweltschutzbewegungen statt.

Von den grössten schweizerischen Firmen wurden nur traditionelle Industrie- 
firmen untersucht. Neuere Firmenkonglomerate, reine Handelsfirmen oder Firmen mit einem steuerpolitisch motivierten Sitz - aber ohne eigentliche Produktion in der Schweiz - wurden nicht berücksichtigt.

Zur besseren Vergleichbarkeit der einzelnen Fallstudien suchten wir nach einer Standardisierung möglichst vieler makro-ökonomischer, naturräumlicher und gesellschaftspolitischer Randbedingungen. Dies wurde erreicht durch eine Konzentration der meisten Fallstudien auf die Grossräume von Bombay und Rio de Janeiro - Sao Paulo. Die beiden Regionen gelten als die am dichtesten industrialisierten Gebiete in Asien bzw, in Lateinamerika und wir fanden in beiden Regionen Tochterfirmen von allen bedeutenden multinationalen Unternehmungen der Schweiz. Zusätzlich zu den Fallstudien von schweizerischen Grosskonzernen untersuchten wir Tochterfirmen von zwei mittelgrossen Schweizer Unternehmen, zwei Tochterfirmen von grösseren deutschen multinationalen Unternehmungen sowie einige lokale indische und brasilianische Firmen. Mit der Analyse dieser Konkurrenzfirmen wollten wir insbesondere den Stand des Umweltmanagements der Firmen unter schweizerischem Einfluss im internationalen Vergleich beurteilen.

\begin{tabular}{|c|c|c|c|}
\hline Unternehmen & Land & Branche & $\begin{array}{l}\text { Eröffnung/ } \\
\text { Erweiterung }\end{array}$ \\
\hline $\begin{array}{l}\text { ABB } \\
\text { ABB } \\
\text { Alusuisse } \\
\text { Attisholz } \\
\text { Chadler } \\
\text { Ciba-Geigy } \\
\text { Ciba-Geigy } \\
\text { F. Hoffmann-La Roche } \\
\text { Holderbank } \\
\text { Holderbank } \\
\text { Laufen } \\
\text { Nestlé } \\
\text { Sandoz } \\
\text { Sandoz }\end{array}$ & $\begin{array}{l}\text { Indien, } \\
\text { Indonesien } \\
\text { Venezuela } \\
\text { Chile } \\
\text { Brasilien } \\
\text { Indien } \\
\text { Brasilien (2) } \\
\text { Indien } \\
\text { Indien } \\
\text { Brasilien } \\
\text { Brasilien (2) } \\
\text { Brasilien (4) } \\
\text { Indien } \\
\text { Brasilien }\end{array}$ & $\begin{array}{l}\text { Maschinen / Energie } \\
\text { Maschinen / Energie } \\
\text { Steine und Erden } \\
\text { Zellstoff } \\
\text { Lebensmittel } \\
\text { Chemie, Agro, Pharma } \\
\text { Chemie, Agro } \\
\text { Pharma } \\
\text { Steine und Erden } \\
\text { Steine und Erden } \\
\text { Steine und Erden } \\
\text { Lebensmittel } \\
\text { Agro, Pharma } \\
\text { Pharma, Agro, Chemie }\end{array}$ & $\begin{array}{l}1990 \\
1986-1988 \\
1980 \\
1994 \\
1954-1980 \\
1972 \\
1981 \\
1960 \\
1995(?) \\
1973-1983 \\
1954-1990 \\
1921-1980 \\
1960 \\
1961\end{array}$ \\
\hline $\begin{array}{l}\text { BASF (Deutschland) } \\
\text { Bayer (Deutschland) }\end{array}$ & $\begin{array}{l}\text { Brasilien } \\
\text { Indien }\end{array}$ & $\begin{array}{l}\text { Pharma, Chemie, Agro } \\
\text { Pharma, Chemie, Agro }\end{array}$ & $\begin{array}{l}1960 \\
1967\end{array}$ \\
\hline Khimline (Indien) & Indien & Maschinen & 1990 \\
\hline $\begin{array}{l}\text { Aracruz (Brasilien) } \\
\text { BAUXIT (Brasilien) } \\
\text { CADAM (Brasilien) } \\
\text { FACEL (Brasilien) } \\
\text { PetroSix (Brasilien) }\end{array}$ & $\begin{array}{l}\text { Brasilien } \\
\text { Brasilien } \\
\text { Brasilien } \\
\text { Brasilien } \\
\text { Brasilien }\end{array}$ & $\begin{array}{l}\text { Zellstoff } \\
\text { Steine und Erden } \\
\text { Steine und Erden } \\
\text { Zellstoff } \\
\text { Fossile Brennstoffe }\end{array}$ & $\begin{array}{l}1968-1990 \\
1973 \\
1975 \\
1974 \\
1986\end{array}$ \\
\hline
\end{tabular}

Abbildung 1: Untersuchte Werke 
Mit den Fallstudien der genannten Firmen werden folgende Kriterien erfüllt:

1. Breites Spektrum in bezug auf die Beteiligungsart der Schweizer Firmen: Insgesamt erhielten wir zu 27 Fallstudien eingehende Unterlagen (18). Zwanzig Betriebe befinden sich durch die Kapital- oder durch die Managementbeteiligung in schweizerischer Kontrolle. Die Art der Beteiligung an den Betrieben ist sehr unterschiedlich (vgl. Abb. 2) (19). Die Beziehungen der untersuchten Firmen reichen von hundertprozentigen Töchtern über Joint Ventures bis hin zu Management- und Lizenzverträgen, ohne eigentliche Kapitalbeteiligungen.

\begin{tabular}{|l|ll|}
\hline Art der Beteiligung & Anzahl \\
\hline Faktisch $100 \%$ Tochter & & 11 \\
Joint Venture $(50: 50)$ & & 1 \\
Minderheitsbeteiligung & & 5 \\
Managementvertrag & & 2 \\
Turn-key-Investition & & 2 \\
\hline
\end{tabular}

Abbildung 2: Art der Beteiligung von schweizerischen Firmen an den Betrieben der Fallstudien

2. Breites Branchenspektrum: Die gewählten Firmen stammen aus der Nahrungsmittel-, der Chemie-, der Metallverarbeitungs-, der Baumaterial- und der Kraftwerksindustrie (20).

3. Unterschiedliches Alter der Produktionsanlagen: Die Zeitachse der untersuchten Fallstudien reicht von über 20 Jahre alten Projekten bis hin zu Projekten, die erst im Bau sind und somit die Standortentscheidung erst kurze Zeit zurückliegt (21).

4. Vergleichbare Konkurrenzfirmen: Um den Stand schweizerischer Umweltschutzmassnahmen international zu vergleichen, wurden in der Nähe der schweizerischen Firmen auch zwei deutsche, eine indische und fünf brasilianische Firmen untersucht.

5. Leicht identifizierbare Umweltprobleme: Aufgrund des hohen Industrialisierungsgrades der Untersuchungsregionen waren die Umweltbelastungen unschwer feststellbar.

\section{Resultate}

\subsection{Motivation zur Direktinvestition}

Um die Frage zu beantworten, ob schweizerische Unternehmungen ökologisch motivierte Standortarbitrage vornehmen, wurden die Firmen in allen Interviews in Indien und in Brasilien nach den Gründen für die Wahl ihres Standortes gefragt. In 
der folgenden Abbildung 3 sind die am häufigsten genannten Motive aufgeführt.

\begin{tabular}{|l|c|c|}
\hline $\begin{array}{l}\text { Gründe für } \\
\text { Produktionsstandort }\end{array}$ & alle Firmen (23) & Schweiz. Firmen (18) \\
\hline Nähe zum Absatzmarkt & 19 & 15 \\
Handelsschranken des & & \\
Gastlandes & 4 & 3 \\
Zugang zu Rohstoffen & 10 & 7 \\
Geringere Produktionskosten & 5 & 5 \\
Geringere Umweltauflagen & 0 & 0 \\
Produktionskosten kein Motiv & 9 & 10 \\
\hline
\end{tabular}

Abbildung 3: Gründe für die Wahl des Produktionsstandortes (22)

Hauptmotive für die Wahl des Produktionsstandortes waren die traditionellen Argumente aus der Theorie der Direktinvestitionen. Die Nähe zu einem potentiellen Absatzmarkt wird in über $80 \%$ der Fälle - zum Teil als primärer Grund - genannt. Einige Betriebe in Indien sind als Antwort auf die indischen Handelsschranken entstanden. Eine ebenfalls sehr wichtige Motivation für eine Investition in einem Entwicklungsland war die Nähe zu Rohstoffen (gegen $40 \%$ ). Insbesondere bei Massengütern findet aus Gründen relativ hoher Transportkosten ein erster Veredelungsschritt oft nahe dem Ort der Rohstoffgewinnung statt. Nur etwa ein Fünftel aller befragten Firmen sehen, wenn auch nie in erster Priorität, günstigere Produktionskosten als Motiv für ihre Standortwahl. In den überwiegenden Fällen spielte die Frage der unterschiedlichen Produktionskosten explizit keine Rolle. Auch wurden in keinem Fall die geringeren Produktionskosten aufgrund von schwachen Umweltschutzauflagen als Motivation genannt.

Weil der Export von Produkten zurück ins Land der Mutterfirma ein gewisses Indiz für eine Standortarbitrage darstellen könnte, befasste sich ein weiterer Fragenkomplex in unseren Interviews mit den Absatzmärkten.

\begin{tabular}{|l|c|c|}
\hline Art der Absatzmärkte & alle Werke (27) & $\begin{array}{c}\text { Schweiz. } \\
\text { Firmen (19) }\end{array}$ \\
\hline Produktion nur für den Binnenmarkt & $45 \%$ & $50 \%$ \\
Produktion auch für Export & $55 \%$ & $50 \%$ \\
Export der Mehrheit der Produktion & $22 \%$ & $5 \%$ \\
Export einzelner Spezialprodukte & $33 \%$ & $45 \%$ \\
Export ins Mutterland & $7 \%$ & $5 \%$ \\
Export in Drittmärkte & $48 \%$ & $45 \%$ \\
\hline
\end{tabular}


Über die Hälfte aller untersuchten Betriebe exportiert Teile ihrer Produktion. Mengenmässig sind hier aber grosse Unterschiede festzustellen. Neun Firmen exportieren nur einzelne arbeitsintensive Spezialprodukte. Nur etwas mehr als ein Fünftel produziert mehrheitlich für den Export. Es handelt sich dabei mit einer Ausnahme um Firmen, die nicht unter schweizerischem Einfluss stehen. Nur zwei Firmen exportieren Produkte in ihr "Heimatland" zurück (23). Im Fall der schweizerischen Firma handelt es sich um einige Spezialchemikalien, die in sehr kleinen Chargen produziert werden.

\subsection{Umweltnormen}

Unsere zweite Frage betrifft die Umweltnormen. Mehrere Konzernleitungen formulieren in ihrem Leitbild eine "One Standard Strategy". Eine solche Strategie impliziert, dass weltweit nach den gleichen (internen) Umweltnormen produziert würde. Auf unsere Fragestellung bezogen, bedeutet dies, dass der Gewinn aus einer ökologisch bedingten Standortarbitrage wegfallen würde. Wir wollten daher im Fall der "One Standard Strategy" von unseren Interviewpartnern wissen, ob sie die lokal geltenden Umweltnormen oder sogar die strengeren schweizerischen Normen im Bereich der Luft- und Abwasserreinhaltung erfüllen. (In Indien gab es zur Zeit unserer Untersuchung noch keine Luftreinhalteverordnung).

\begin{tabular}{|l|ll|}
\hline Betrieb erfüllt schweizerische Umweltnormen & & 0 \\
Betrieb unterschreitet einige lokale Grenzwerte & & 15 \\
$\begin{array}{l}\text { Betrieb erfüllt alle lokalen Umweltnormen } \\
\text { Betrieb profitiert von einigen Übergangsfristen }\end{array}$ & & 1 \\
\hline $\begin{array}{l}\text { Keine Massnahmen zur Luftreinhaltung } \\
\text { implementiert }\end{array}$ & & 3 \\
$\begin{array}{l}\text { Betrieb erfüllt Normen der Abfallbewirtschaftung } \\
\text { nicht }\end{array}$ & & 4 \\
Betrieb erfüllt Abwassernormen nicht & & 3 \\
\hline
\end{tabular}

Abbildung 5: Erfüllen der Umweltnormen durch die Tochterfirmen

Kein besuchter Betrieb erfüllt die Umweltnormen, wie sie vom Gesetzgeber in der Schweiz verlangt werden. Die Implementierung einer konsequenten "One Standard Strategy" ist in keinem Unternehmen vollzogen. Hingegen finden sich in den meisten Fallstudien umweltrelevante Investitionen, die über das vom lokalen Gesetzgeber vorgeschriebene Mass hinausgehen. Verschiedene Begründungen werden für ein solches Verhalten angegeben: 
1. Viele umweltrelevante Investitionen betreffen neue Technologien, die primär der Verbesserung der ökonomischen Effizienz dienen und nur sekundär zu einer erhöhten Umweltverträglichkeit der Produktion führen.

2. Eine weitere Motivation für freiwillig weitergehende Investitionen zur Schonung natürlicher Ressourcen ergibt sich aus der Antizipation zukünftiger umweltpolitischer Massnahmen innerhalb des Investitionsraumes. Da gesetzliche Übergangsbestimmungen nur kurze Zeit gültig sind, kann es betriebswirtschaftlich sinnvoll sein, eine Anlage frühzeitig den zu erwartenden Bedingungen anzupassen.

3. Umweltschutzmassnahmen sind oft zugleich Massnahmen im Bereich der Public Relations. Die Firmen sind sich bewusst, dass es in der heutigen Zeit nicht nur darum geht, Konsumenten und Aktionäre davon zu überzeugen, dass die Firma den neuesten ökologischen Anforderungen genügt, sondern dass vielmehr zwei weitere Faktoren dazugekommen sind: Durch eine grössere Gewichtung der umweltverträglichen Produktion soll die Industrie oder eine Unternehmung als Arbeitgeber attraktiv bleiben, und in der Öffentlichkeit soll ein Klima des Vertrauens geschaffen werden (24).

\subsection{Umweltschutzkosten (25)}

Unsere dritte Frage befasste sich mit den Kosten, die durch den betrieblichen Umweltschutz induziert werden. Es handelt sich dabei z.B. um Energie- und Unterhaltskosten für Entsorgungsanlagen, um Kapitalkosten für Umweltschutzinvestitionen, um Kosten für die Forschung und Entwicklung von umweltverträglicheren Produkten und Produktionsprozessen sowie um Personalkosten in der Umweltabteilung. Genaue Angaben über die Grössenordnung dieser Kosten haben wir aber nur von sehr wenigen Betrieben (aus der chemischen Industrie) erhalten. Die folgende Abbildung 6 zeigt den durchschnittlichen Anteil der Umweltschutzausgaben am Gesamtaufwand für die untersuchten chemischen Firmen in der Schweiz, in der BRD und in Indien.

Aufwand in der Schweiz

Aufwand in der BRD

Aufwand in Indien

$3 \%$

$5 \%$

$1 \%$

Abbildung 6: Durchschnittlicher Umweltschutzkostenanteil am Gesamtproduktionsaufwand in der chemischen Industrie

Besser war die Datenlage in Bezug auf die Grössenordnung von Investitionen in Umwelttechnologien. Auf den betrieblichen Umweltschutz fielen in den untersuchten Werken zwischen 1\% und 6\% der Gesamtinvestitionen (26). Im Durch- 
schnitt der siebzehn Werke, die monetäre Angaben über ihre Umweltschutzinvestitionen machen konnten, ergibt dies einen Anteil von 4,2\% des Gesamtinvestitionsvolumens. Dieser Prozentsatz soll in den kommenden Jahren höher liegen, da in einigen Werken die Absicht besteht, im Bereich des Umweltschutzes "aufzurüsten". Die grössten Umweltschutzinvestitionen, absolut und im Verhältnis zu den Gesamtinvestitionen, fanden wir in der Zement- und Keramikindustrie. Bezeichnenderweise sind es gerade diese Werke, die innert sehr kurzer Zeit einen grossen Nutzen aus ihren Massnahmen für den Umweltschutz ziehen können. Drei Viertel ihres betrieblichen Umweltschutzes sind für die Installation von sehr kapitalintensiven Elektrofiltern bestimmt. Mit diesen Filtern werden nicht nur Natur und Arbeiter vor feinem Staub verschont, sondern es können riesige Mengen an wertvollen Rohstoffen zurückbehalten werden (27).

Für die Schweiz ist noch keine Untersuchung bekannt, die repräsentative Auskunft über den durchschnittlichen Anteil der industriellen Umweltschutzmassnahmen an den totalen jährlichen Investitionen gibt. Aus unserer Analyse von Dokumenten können wir z.T. die entsprechenden Zahlen abschätzen. Diese liegen je nach Branche zwischen $3 \%$ und $15 \%$ (28).

\section{Diskussion und Zusammenfassung}

In verschiedenen theoretischen und empirischen Abhandlungen findet sich immer wieder die These, dass unterschiedliche Umweltnormen die internationale Faktorallokation zwischen industrialisierten Staaten und weniger entwickelten Ländern beeinflussen. Wir untersuchten für 10 Schweizer Grossunternehmen mit Produktionsstätten in weniger entwickelten Ländern, ob der Zwang zu immer neuen Umweltschutzmassnahmen in der Industrie eine ausreichende Motivation darstellt, Teile der Produktion an einen Standort mit geringeren Umweltauflagen und/oder kaum existierendem Vollzug zu verlagern.

Unsere Analyse zeigt, dass die Konzerne tatsächlich mit verschiedenen Umweltstandards produzieren. Dieses Resultate steht im Widerspruch zu den Leitbildern der meisten der untersuchten Firmen, demgemäss eine ökologische 'One Standard Strategy' anzustreben sei; denn verschiedene Umwelt-Standards bedeuten für eine Firma nicht nur, dass Umweltschutzaufwendungen eingespart werden können, sondern auch dass neue Kosten entstehen können:

1. Unterschiedliche umwelttechnische Standards bedingen oft auch unterschiedliche Produktionsprozesse. Die meisten der untersuchten Konzerne haben ein internes Kontrollsystem, mit dem die Effizienz der Produktion konzernweit überprüft und verglichen wird. Diese Überprüfung der Tochterunternehmen ist im Falle verschiedener Produktionsprozesse mit einem weit grösseren Aufwand verbunden als im Falle von einheitlichen Produkten und Produktionsstandards.

2. In einer Welt mit sehr hoher Informationsdiffusion wird in der Öffentlichkeit nicht mehr unterschieden, ob ein Umweltproblem bei einer Tochterfirma oder beim 
Mutterhaus entsteht. Ein angeschlagenes Image wirkt sich auf den Absatz-, den Kapital- und den Arbeitsmarkt negativ aus (Imagekosten).

3. Eine weitere Motivation für freiwillige Investitionen um natürliche Ressourcen zu schonen ergibt sich aus der Antizipation zukünftiger umweltpolitischer Massnahmen innerhalb des Investitionsraumes. Wird es unterlassen, eine Anlage im Rahmen des üblichen Rhythmus für Ersatzinvestitionen frühzeitig den zu erwartenden Bedingungen anzupassen, können aufgrund der oft sehr knappen gesetzlichen Übergangszeiten bei neuen Umweltschutzgesetzen höhere Adaptionskosten entstehen.

Unsere Analyse zeigt, dass sich die Differenzen im betrieblichen Umweltschutz innerhalb der Konzerne tendenziell eher verkleinern, und dass aus diesem Grund ein potentieller Vorteil einer ökologisch bedingten Standortarbitrage verkleinert wird. Direktinvestitionen von schweizerischen multinationalen Unternehmungen in weniger entwickelten Ländern waren in allen Fällen hauptsächlich durch die klassischen Motive wie Zugang zum Markt oder zu den Rohstoffen bedingt. Unterschiedliche Produktionskosten spielten generell eine untergeordnete Rolle. Die Begründung dieser Aussage liegt darin, dass den niedrigeren Umweltschutzkosten in einem weniger entwickelten Land eine ganze Reihe von Kostenfaktoren gegenüberstehen, welche die genannten Einsparungen zum Teil überkompensieren. Transaktionskosten, höhere Kapitalkosten, Ausbildungskosten für technische Angestellte, kleinere Grösseneffekte (economics of scale) sind einige dieser zusätzlichen Aufwendungen.

Konkret ergab die Auswertung unserer Befragungen bei Produktionsanlagen in Entwicklungsländern einen Investitionsanteil für Umweltschutzmassnahmen zwischen 1 bis $6 \%$ des Gesamtinvestitionsvolumens. Verglichen mit den entsprechenden Kosten in der Schweiz ergibt sich eine Differenz (Kosteneinsparung) von etwas mehr als 5\%. Werden in mittlerer Zukunft die Umweltschutzbestimmungen in den Entwicklungsländern ebenfalls strenger, so wird ein Betrieb bestenfalls für einen beschränkten Zeitraum mit Einsparungen rechnen können. Diese Differenz stellt damit allein keine genügend grosse Motivation dar, um Teile der Produktion an einen Standort in einem Entwicklungsland zu verlagern.

Zusammenfassend zeigt sich, dass aufgrund der Resultate unserer Falluntersuchung die Existenz einer ökologisch bedingten Standortarbitrage bei schweizerischen Unternehmen zu verneinen ist.
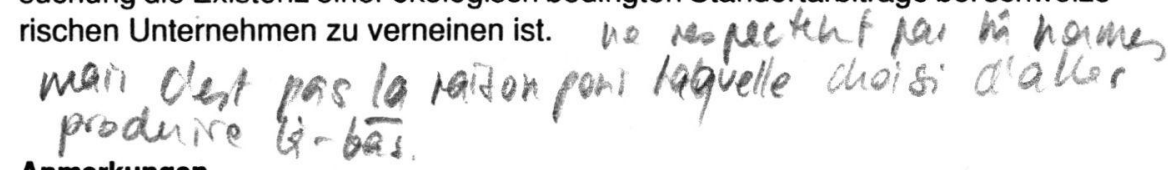

\section{Anmerkungen}

1. Die OECD (1992) gibt eine gute Übersicht über die Entwicklung der Direktinvestitionen in den siebziger und achtziger Jahren.

2. Zur Theorie der multinationalen Unternehmungen als Form internationaler Faktorallokation seien die Arbeiten von Casson (1990) und Caves (1982) hervorgehoben.

3. Castelmann (1979), Jaafar (1989), Leonard (1985) und Mayo (1989), Schreiber und Schreiber (1987). 
4. Anandalingam und Westfall (1987), Andersson (1991), Knödgen (1982), Rogerson (1990), Staffort (1985), Tobey (1990) und UNCTC (1985) sind weitere der uns bekannten empirischen Untersuchungen. Rauscher (1993) suchte mit Hilfe von Modellen eine Evidenz für ein Öko-Dumping in weniger entwickelten Staaten; er nennt auch die theoretische Literatur, die sich in den achtziger Jahren mit dieser Fragestellung beschäftigt hat.

5. Blazejczak (1993), Heerings (1993), Low und Yeats (1992), Lucas et al. (1992).

6. 1992 wurden in der Schweiz drei unabhängige Studien zu dieser Fragestellung veröffentlicht. Die vorliegende Darstellung basiert auf unserer eigenen Untersuchung (Wyss und Straubhaar 1991; Wyss 1992). Unsere Resultate werden aber durch die Studien von Brugger und Maurer (1992) und Burkhard (1992) bestätigt.

7. Nach Borner et al.(1990: 109).

8. Michael Porter (1990: $80 \mathrm{ff})$ vergleicht verschiedene Industriestandorte bezüglich ihrer Ausstattung mit Produktionsfaktoren. Die Verfügbarkeit der Produktionsfaktoren (und damit die Kosten für deren Beschaffung) beeinflussen seiner Meinung nach massgebend die Effizienz ihrer Nutzung. Sind Produktionsfaktoren an einem Standort relativ knapp, werden die Produktionsbetriebe motiviert, mittels Innovationen die Effizienz der Faktorverwendung zu erhöhen.

9. Arbitrage wird von Bender (1977) definiert als "die auf Gewinnmaximierung oder Kostenminimierung gerichtete Ausnutzung der im gleichen Zeitpunkt auf mindestens zwei Teilmärkten für ein homogenes Gut existierenden Preisunterschiede". Der Begriff 'Arbitrage' wurde ursprünglich vor allem für eine Ausnutzung von Marktunvollkommenheiten auf den internationalen Finanzmärkten verwendet. Die Zielsetzung, aus interlokativen Kostenunterschieden zu profitieren, wurde später auch auf die Standortwahl von Industriebetrieben übertragen. Siebert (1989: 189) weist zum Beispiel in seinem Artikel über aussenwirtschaftliche Aspekte der europäischen Integration darauf hin, dass unterschiedliche Umweltregelungen der europäischen Staaten zu einem Anreiz von Standortarbitrage führen können.

10. Schreiber und Schreiber (1987) zeigen Beispiele von japanischer Standortarbitrage auf den Phillipinen, und Jaafar (1985) untersuchte solche in Indonesien. Beide Berichte kommen zum Schluss, dass Verlagerungen aufgrund der strengen Umweltregulierungen in Japan sehr wohl stattgefunden haben. Illi (1991), Germund (1990) und Siddharthan und Kumar (1990) zeigen, dass weltweite Differenzen bei den Umweltschutzkosten ein möglicher Grund für die weltweite, interne Arbeitsteilung innerhalb von amerikanischen Unternehmungen sind.

11. Für eine feinere Aufgliederung der Funktionen der Umweltfaktoren im Produktionsprozess siehe Siebert (1990).

12. Die verschiedenen Gründe werden u.a. im Sammelband "Mit Ökonomie zur Ökologie" von Frey et. al. (1992) aufgearbeitet.

13. Beyer (in Hauff 1992: 245-260) gibt einen systematischen Überblick über die Vielzahl von Instrumenten, die heute in der Umweltpolitik westlicher Staaten diskutiert werden.

14. Einen umfassenden Überblick der Breite von Interdependenzen zwischen Umweltpolitik und Aussenwirtschaftspolitik bietet der Sammelband von Anderson und Blackhurst (1992).

15. Walter und Ugelov (1979) fragen sich in ihrem vielzitierten Artikel, ob in gewissen Entwicklungsländern sogenannte "Verschmutzungs-Häfen" für emissionsintensive Industrien entstünden. Pearson (1987) zitiert weitere Vorwürfe an die Adresse einzelner Entwicklungsländer, dass sie Öko-Dumping betreiben, um der lokal produzierenden Industrie einen komparativen Vorteil gegenüber der ausländischen Konkurrenz zu verschaffen. Modelle der theoretischen Untersuchungen (z.B. von Rauscher 
1993) zeigen hingegen, dass mit Öko-Dumping längerfristig kaum eine effiziente Förderung der Industrie erreicht werden kann.

16. Zur Umweltrelevanz von nicht industriellen Projekten in weniger entwickelten Ländern gibt die Studie von Messerli et al. (1987) einen wertvollen Überblick.

17. Insbesondere soll die ganze Diskussion um die sozio-ökologische Relevanz der Anwendung von Agrochemikalien oder der Vermarktung von Kindernahrungsmitteln in Entwicklungsländern ausgeklammert bleiben.

18. Davon konnten 21 Werke persönlich während einem oder mehrerer Tage besucht werden. Für die zwei im Bau befindlichen Werke in Chile und Indien sowie für weitere vier bestehende Werke in Indonesien, Indien (2) und Venezuela wurden die Informationen aus Dokumenten (z.B. Machbarkeitsstudien und Umweltverträglichkeitsberichte) und durch Interviews in der Schweiz beschafft.

19. Bei vielen Betrieben ist die kapitalmässig höchstmögliche Beteiligung für ausländische Partnerfirmen im Gesetz festgelegt. So dürfen z.B. in Indien ausländische Firmen nur Minderheitsbeteiligungen an indischen Firmen besitzen. In zwei weiteren Fällen besteht nur eine symbolische Minderheitsbeteiligung. Diese Betriebe werden oder wurden aber dennoch über Managementverträge durch schweizerische Wirtschaftssubjekte gelenkt. Unklar ist die Möglichkeit der schweizerischen Einflussnahme (z.B. auf den Umweltschutz) bei den Projekten, die schlüsselfertig (Turn-key-Investition) durch eine schweizerische Firma erstellt werden, deren Management nachher aber an eine lokale (staatliche) Firma übergeht. Hier definiert zwar einerseits der Auftraggeber in den 'Terms of Reference' welche technischen Eigenschaften erfüllt sein müssen, andererseits kann der Projektleiter mit seiner beratenden Tätigkeit mindestens die Notwendigkeit von Umweltschutzmassnahmen aufzeigen.

20. Nicht berücksichtigt ist die Textilindustrie. Keine, der von uns angefragten Firmen war bereit, an der Untersuchung teilzunehmen.

21. Viele Werke wurden bereits in den sechziger und siebziger Jahren gebaut. Der erste Investitionsentscheid fand also in einer Zeit statt, als Umweltschutzfragen einzig im Bereich des Gewässerschutzes in Europa aktuell waren. Dennoch mussten auch in jüngster Zeit im Zusammenhang mit zum Teil namhaften Erweiterungen immer wieder Investitionsentscheide gefällt werden. Auf der anderen Seite hat z.B. Roche Ende 1993 beschlossen, die Minderheitsbeteiligung an einem von uns besuchten Werk in Indien zu verkaufen.

22. Es waren Mehrfachnennungen möglich. Wir berücksichtigen für diese Tabelle nur jene Betriebe, deren Mutterfirma zur Zeit des Investitionsentscheides ausserhalb des Standortlandes lag. Die drei Firmen CADAM, Bauxit und FACEL, die heute in brasilianischem Besitz sind, waren zur Zeit der Standortwahl in amerikanischem Besitz und werden daher unter "gesamt" berücksichtigt. Nicht berücksichtigt ist hingegen die Firma Chadler, die erst seit 4 Jahren mehrheitlich in schweizerischem Besitz ist und zur Zeit der Gründung rein brasilianisch war.

23. Siddharthan und Kumar (1990) analysierten den Handel innerhalb von amerikanischen mutinationalen Unternehmungen. Aus ihrer Analyse schliessen sie, dass die international unterschiedlichen Umweltnormen einen Grund für den Intra-Firmenhandel darstellen.

24. Abbildung $\mathbf{5}$ darf nicht darüber hinwegtäuschen, dass ein grösserer Teil der untersuchten Betriebe, gegenüber den lokalen, zur Zeit der Untersuchung geltenden Emissionsgrenzwerten im Verzug waren. Zum Teil waren diese Normen recht jung. Für bestehende Firmen war eine Übergangszeit vorgesehen. Nur in einem Fall war diese bereits abgelaufen und der Firma drohte kurz nach unserem Besuch aus ökologischen Gründen der Entzug der Betriebsgenehmigung. 
25. Bei der Beurteilung der betrieblichen Finanzdaten musste eine nicht unwesentliche Fehlerquelle berücksichtigt werden. Die Standorte der meisten Fallstudien liegen in Ländern mit einer hohen Inflationsrate. Die damit verbundenen hohen Schwankungen der Wechselkurse zum Dollar und die zeitweiligen Währungsreformen führen zu Schwierigkeiten beim Vergleich der Rechnungslegung der einzelnen Firmen. Bei der Interpretation der Zahlen musste daher berücksichtigt werden, dass die Zahlen in den Geschäftsberichten zum Teil nach unterschiedlichen Konsolidierungsgrundsätzen ausgewiesen werden.

26. Die Investitionsvolumina waren sehr unterschiedlich, da es im Ermessen der Betriebe liegt, ob sie Investitionen dem Umweltschutz zuordnen oder nicht. Oft werden auch Massnahmen zur Erhöhung der Produktionssicherheit dem Konto Umweltschutz zugeordnet. Im übrigen hängen die unterschiedlichen Anteile der Umweltschutzinvestitionen mit der Vielfalt der untersuchten Betriebe zusammen. Der kleinste Betrieb, den wir besucht haben, hat eine Investitionssumme von einigen wenigen Mio. SFr.; das grösste Projekt band nach drei Jahren Bauzeit 1,7 Mrd. SFr. flüssige Mittel und Fremdkapital. Wir können aber davon ausgehen, dass die durchschnittliche Gesamtinvestitionssumme unserer Fallstudien zwischen 80 und 180 Mio. SFr. liegt.

27. Noch nicht einbezogen in unsere Rechnungen ist der hohe Anteil geplanter Umweltschutzinvestitionen in einem der beiden erst im Bau befindlichen Werke. Die Investoren wollen ein sehr günstiges Werk bauen, ohne aber mit Umweltschutzmassnahmen zu sparen. In Antizipation bald enstehender Umweltgesetze werden daher gegen $15 \%$ der Investitionssumme für den betrieblichen Umweltschutz eingesetzt.

28. Die breite Erfassung des deutschen statistischen Bundesamtes (1991) errechnet für die BRD einen durchschnittlichen Anteil der Umweltschutzinvestitionen am Gesamtinvestitionsvolumen von 4,5 \% - 5,2 \%. Diese Schätzung deckt sich mit den Ergebnissen des Vereins Schweizerischer Maschinenindustrieller (VSM). Demzufolge dienten anfangs der neunziger Jahre etwa 7\% der jährlichen Investitionen primär dem Erhalt der Umwelt (NZZ 1992). Hierbei liegt der Investitionsschwerpunkt bei der Luftreinhaltung (Abluftreinigung) und beim Gewässerschutz - ein deutliches Beleg dafür, dass Umweltschutzinvestitionen gesetzesinduziert erfolgen (NZZ 1992).

\section{Literatur}

Altmann, J, 1992: Das Problem des Umweltschutzes im internationalen Handel. H. Sauter (Hrsg.): Entwicklung und Umwelt: 207-244. Duncker \& Humblot, Berlin

Anandalingam, G., Westfall, M., 1987: Hazardous Waste Generation and Disposal: Options for Development Countries. Natural Resources Forum 11 (1): 37-47

Anderson, K., Blackhurst, R., 1992: Greening World Trade Issues. Harvester \& Wheatsheaf, New York

Andersson , Th., 1991: Multinational Firms and Pollution in Developing Countries. C. Folke und T. Kaberger (Hrsg.): Linking the Natural Environment and the Economy. Kluwer Academic Publishers, Dordrecht

Bender, D., 1977: Handbuch der Wirtschaftswissenschaft. Fischer, Stuttgart.

Blazejczak, J., Kohlhaas, M., Seidel, B., Trabold-Nübler, H., 1993: Umweltschutz und Industriestandort. Berichte 1 (93) des Umweltbundesamtes. Erich Schmidt, Berlin

Borner, S., Brunetti, A., Straubhaar, T., 1990: Die Schweiz AG, Vom Sonderfall zum Sanierungsfall? Neue Zürcher Zeitung, Zürich.

Brugger, E.A., Maurer, M., 1992: Privatwirtschaftlicher Transfer von Ökologie-know-how in Entwicklungsländer. unpubl. Schlussbericht im NFP 28, Schweizer Nationalfonds, Bern 
Brugger, E.A., Messerli, B., Straubhaar, Th., Wyss, M., 1993: Schwarze Schafe oder Weisse Ritter? Zur Öko-Effizienz multinationaler Unternehmungen in Entwicklungsländern. Geographica Bernensia, Bern

Buckley, P.J., (Hrsg.) 1992: New Directions in International Business. Elgar, Aldershot Burkhard, P., 1992: Umweltverhalten von Multis. Strukturberichterstattung. Bundesamt für Konjunkturfragen, Bern

Casson, M., 1990: Multinational Corporations. Elgar, Aldershot

Castelmann, B.I., 1979: The Export of Hazardous Factories to Developing Nations. International Journal of Health Services 9 (4): 569-606

Cropper, M.L., Oates, W.E., 1992: Environmental Economics: A Survey. Journal of Economic Literature 30: 675-740

Dunning, J.H., 1981: International Production and Multinational Enterprise. London

Frey, R.L., Staehelin-Witt, E., Blöchliger, H., (Hrsg.) 1991: Mit Ökonomie zur Ökologie. Helbling und Lichtenhahn, Basel

Germund, W., 1990: Mexikos Nordgrenze verwandelt sich zunehmend in eine Kloake. Basler Zeitung 197: 18

Hauff, M. von, Schmid, U., (Hrsg.) 1992: Ökonomie und Ökologie. Schäffer-Poeschel, Stuttgart

Heerings, H., 1993: The Role of Environmental Policies in Influencing Patterns of Investments of Transnational Corporations, OECD, (Hrsg) Environmental Policies and Industrial Competitiveness, Paris

Houde, M.F., 1992: Foreign Direct Investment. OECD Observer 176 (4)

IIIi, L., 1991: EG 92: Wohlstand in Europa - Armut in der Dritten Welt. Partnerschaft 123: 9 Jaafar, A. B., 1985: Foreign Investment, Trade, Incentives and Disincentives of Pollution Control. unpubl. Paper from the Department of Environment, Malaysia

Knödgen, G., 1982: Umweltschutz und industrielle Standortentscheidung. Campus, Berlin Leonard, J., 1985: Pollution and the Struggle for the World Product. Cambridge University Press, Cambridge

Low, Patrick and Alexander. Yeats (1992), Do "Dirty" Industries Migrate? Patrick Low (ed.) International Trade and the Environment, Worldbank Discussion Paper 159 : 89-103 Lucas, Robert, E.B., David Wheeler and Hemamala Hettige (1992), Economic Development, Environmental Regulations and the International Migration of Toxic Industrial Pollution: 1960-88. Patrick Low (ed.) International Trade and the Environment, Worldbank Discussion Paper $159: 67-87$

Mayo, E., 1990: 1992: European Wealth, Third World Poverty? A WDM-Report. World Development Movement, London

Messerli, B., Bisaz, A., Kienholz, H., Winiger, M., 1987: Umweltprobleme und Entwicklungszusammenarbeit. Reihe P 16. Geographica Bernensia, Bern

NZZ, 1992: Mehr Umweltinvestitionen der Maschinenindustrie. Neue Züricher Zeitung (NZZ). 301:23

OECD (1992), Globalisation of Industrial Activities, Paris

Pearson, S.Ch., 1987: Multinational Corporations, Environment and the Third World, Duke University Press, Durham

Pethig, R., 1976: Pollution, Welfare, and Environmental Policy in the Theory of Comparative Advantage. Journal of Environmental Economics and Management 2: 160-169

Porter, M., 1990: The Competitive Advantage of Nations. Macmillan Press, London

Rauscher, M., 1993: On Ecological Dumping. Nota di Lavoro 29.93, Fondazione ENI Enrico Mattei, Milano.

Rogerson, C.M., 1990: Environmentally Hazardous Industry in South Africa: A Spatial View. GeoJournel 22 (3): 321-328 
SBG (Schweizerische Bankgesellschaft), 1991: Die 100 grössten Firmen in der Schweiz. SBG, Zürich

Schmidheiny, S., (Hrsg.) 1992: Kurswechsel. Globale unternehmerische Perspektiven für Entwicklung und Umwelt. Atlantis und Winkler, München

Schreiber, H., Schreiber, V., 1987: Environmental Consequences of Transnational Corporation Activities in Developing Countries: The Case of the Philipines. Institute of Environmental Policy, Bonn

Siddharthan, N.S., Kumar, N., 1990: The Determinants of Inter-Industry Variations in the Proportion of Intra-Firm Trade: The Behavier of US Multinationals. Weltwirtschaftliches Archiv 126: 581-591

Siebert, H., 1977: Environmental Quality and the Gains from Trade. Kyklos 30: 657-673 Siebert, H., 1989: Perspektiven zur Vollendung des europäischen Binnenmarktes. Kyklos 42: 181-201

Siebert, H., 1990: Natürliche Ressourcen und Weltwirtschaft. Weltwirtschaftliches Archiv 126: $1-24$

Siebert, H., 1991: Aussenwirtschaft. UTB 212. Fischer, Stuttgart

Stafford, H.A. 1985: Environmental Protection and Industrial Location. Annals of the Association of American Geographers 75 (2): 227-240

Statistisches Bundesamt, 1991: Investitionen für Umweltschutz im produzierenden Gewerbe 1989. Stuttgart

Straubhaar, Th., Wyss, M., 1992: Ökologische Produktion kennt keine Landesgrenze. Entwicklung/Développement, (DEH/BAWI) 36: 24-26

Strubel, M., 1992: Internationale Umweltpolitik. Leske, Opladen.

Tobey, J.A., 1990: The Effects of Domestic Environmental Policies on Patterns of World Trade: An empirical Test. Kyklos 43: 191-209

UNCTC, 1985: Environmental Aspects of the Activities of Transnational Corporations: A Survey. New York

Walter, I., 1976: Studies in International Environmental Economics. Wiley, New York

Walter, I., Ugelow, J., 1979: Environmental Policies in Developing Countries. Ambio 8: $102-$ 109

WCED 1987: Our Common Future. Oxford University Press, Oxford.

Weltbank, 1992: Weltentwicklungsbericht 1992. 'Entwicklung und Umwelt'. UNO Verlag, Bonn

Wyss, M., 1992: Ökologische Aspekte der wirtschaftlichen Zusammenarbeit mit Entwicklungsländern. Reihe P 27. Geographica Bernensia, Bern

Wyss, M., Straubhaar, Th., 1991: Environmental Arbitrage and the Location of Industrial Plants in Third World Countries. Proceedings of the int. Congress "Innovation, Industrial Progress and Environment, getting ready for the XXIst Century" in Strassburg 


\section{Résumé}

Peut-on affirmer que les règlementations visant la protection de l'environnement incitent les entreprises suisses à déplacer la production vers des pays du Tiers Monde? Les auteurs ont cherché des éléments de réponse à l'aide d'une enquête menée auprès d'une dizaine de filiales de grandes entreprises suisses établies dans les Tiers Monde. Il en résulte que le facteur en question n'a joué aucun rôle dans la décision de créer ces filiales. La motivation de l'investissement se trouve par contre en général dans les facilités d'accès soit au marché local soit à des matières premières. Cela n'empêche que pour ces entreprises et proportionnellement au total des dépenses, le coût de protection de l'environnement est dans le Tiers Monde plus faible qu'en Suisse. Il émerge aussi que les normes internes adoptées par ces entreprises en matière de protection de l'environnement peuvent différer selon les pays. 